\title{
Unit of Electricity and Magnetism
}

National Cancer Institute

\section{Source}

National Cancer Institute. Unit of Electricity and Magnetism. NCI Thesaurus. Code C67319.

A unit of measurement of a property of the electromagnetic interaction. 